\title{
Matrix Metalloproteinase
}

National Cancer Institute

\section{Source}

National Cancer Institute. Matrix Metalloproteinase. NCI Thesaurus. Code C18164.

Matrix metalloproteinases are $\mathrm{Zn}(2+)$-binding endopeptidases that degrade components of the extracellular matrix. These enzymes are implicated in tissue remodeling processes, wound healing, angiogenesis, and tumor invasion. Matrix metalloproteinases have been associated with the development of advanced-stage cancer via their role in tumor progression, invasion, and metastasis. 